\title{
ON THE DIRECT-PRODUCT OF OPERATOR ALGEBRAS I
}

\author{
TAKASI TURUMARU \\ (Received June 16, 1952)
}

1. Introduction. Recently, $\mathrm{R}$ Schatten and J.von Neumann have defined" the direct-product of Banach spaces, and obtained many interesting results - for example, they have proved that the double conjugate space of Banach space of all completely continuous operators on the Hilbert space coincides with the space of all bounded operators on that space (cf. [4: Theorem 5.15]).

As they say, the direct-product of Banach spaces is seen to be an effective tool in dealing with Banach spaces whose elements are operators on some Banach space. On the other hand, from the algebraic standpoint, the direct-product of Banach spaces is considered to be a generalization of the Kronecker product of vector spaces to the infinite dimensional cases; so the author suppose that it is significant to consider the direct-product of the $C^{*}$-algebras as a generalization of the Kronecker product of rings to the infinite dimensional cases.

In the present paper, we shall define the direct-product of operator algebras as Kronecker product of rings, and introduce a suitable norm in this product space; and finally completing this ring by using the norm defined above, we shall construct a new $C^{*}$-algebra as the direct-product of $C^{*}$-algebras.

In $\S 2$, we recall $\mathrm{R}$. Schatten-J. von Neumann's definition of the directproduct and define the product of expressions. In $\S 3$, we consider about the states on the direct-product and introduce a suitable norm (cross-norm in $R$. Schatten's sense) on this direct-product ring. Finally in $\S 4$, we apply the above consideration to the commutative $C^{*}$-algebras, and prove that directproduct of the commutative $C^{*}$-algebras of all continuous functions defined on compact Hausdorff spaces $\Omega$ and $\Gamma$, is isometrically isomorphic to the $C$ *algebra of all continuous functions defined on the product space $\Omega \times \Gamma$; this result may justify our norm, and is related to Dunford-Schatten's results $[1]$.

I will express my hearty thanks to Prof. M. Nakamura for his many valuable suggestions.

2. Definitions and notations. We begin with recalling Schatten-J. von Neumann's definitions and notations.

For any $C^{*}$-algebras $A_{1}, A_{2}$ (with unit), let $X$ be a set of all formal "expressions"

$$
\sum_{i=1}^{n} x_{i} \times y_{i}=x_{1} \times y_{1}+\ldots+x_{n} \times y_{n}
$$


where $x_{i} \in A_{1}, y_{i} \in A_{2}, \quad i=1,2, \ldots, n ; n=1,2, \ldots$.

In $X$, we introduce a relation $\sim$ subjects to the following rules :

(i) $\sum_{i=1}^{n} x_{i} \times y_{i} \sim \sum_{i=1}^{n} x_{p(i)} \times y_{p(i)}$, where $p(1), ., \ldots, p(n)$ denotes any permutation of the integers $1,2, \ldots, n$.

(ii ) $\left(x_{1}^{\prime}+x_{1}^{\prime \prime}\right) \times y_{1}+\sum_{i=2}^{n} x_{i} \times y_{i} \sim x_{1}^{\prime} \times y_{1}+x_{1}^{\prime \prime} \times y_{1}+\sum_{i=2}^{n} x_{i} \times y_{i}$.

(ii') $\quad x_{1} \times\left(y_{1}^{\prime}+y_{1}^{\prime \prime}\right)+\sum_{i=2}^{n} x_{i} \times y_{\iota} \sim x_{1} \times y_{1}^{\prime}+x_{1} \times y_{1}^{\prime \prime}+\sum_{i=2}^{n} x_{i} \times y_{i}$.

(iii) $\sum_{i=1}^{n}\left(a_{i} x_{i}\right) \times y_{i} \sim \sum_{i=1}^{n} x_{i} \times\left(a_{i} y_{i}\right)$, where $a_{i}$ denotes any scalar.

And finally, two expressions $\sum_{i=1}^{n} x_{i} \times y_{i}$ and $\sum_{j=1}^{m} s_{j} \times t_{j}$ in $X$ will be termed equivalent if one can be transformed into the other by a finite number of successive applications of rules (i)-(iii); and we write

$$
\sum_{i=1}^{n} x_{i} \times y_{i} \simeq \sum_{i=1}^{m} s_{j} \times t_{j}
$$

Then we can easily verify that the relation $\simeq$ is reflexive, symmetric, transitive; so we define the linear set $A_{1} \odot A_{2}$ as a set of residue classes of $X$ by this relation $\simeq$.

Now, we define the product and involution $*$ in $X$ as follows :

(product) $\left(\sum_{i=1}^{n} x_{i} \times y_{i}\right) \cdot\left(\sum_{i=1}^{m} s_{j} \times t_{j}\right)=\sum_{i=1}^{n} \sum_{j=1}^{m} x_{i} s_{j} \times y_{i} t_{j}$,

(involution)

$$
\left(\sum_{i=1}^{n} x_{i} \times y_{i}\right)^{*}=\sum_{i=1}^{n} x_{i}^{*} \times y_{i}^{*}
$$

Then the following Lemma can be proved:

LEMMA 1. The product, and the involution* defined above are invariant under the relation $\simeq$. that

Proof. To complete the proof of this lemma, it is [sufficient to show

$$
\sum_{i=1}^{n} x_{i} \times y_{\iota} \simeq \sum_{i=1}^{n^{\prime}} x_{i}^{\prime} \times y_{\iota}^{\prime}, \quad \text { and } \sum_{j=1}^{m} s_{j} \times t_{j} \simeq \sum_{j=1}^{n^{\prime}} s_{j}^{\prime} \times t_{j}^{\prime}
$$

imply

and

$$
\sum_{i=1}^{n} \sum_{i=1}^{m} x_{i} s_{j} \times y_{i} t_{j} \simeq \sum_{i=1}^{n^{\prime}} \sum_{j=1}^{m^{\prime}} x_{i}^{\prime} s_{j}^{\prime} \times y_{i}^{\prime} t_{j}^{\prime},
$$




$$
\sum_{i=1}^{n} x_{i}^{*} \times y_{i}^{*} \simeq \sum_{i=1}^{n^{\prime}} x_{i}^{\prime *} \times y_{i}{ }^{*}
$$

Firstly, by [4: Lemma 1.1] we can assume, without loss of generality, that the sets $\left\{x_{i}\right\},\left\{y_{i}\right\}, \ldots,\left\{s_{j}^{\prime}\right\}$ and $\left\{t_{j}^{\prime}\right\}$ are linearly independent respectively, then $n=n^{\prime}$ and $m=m^{\prime}$ by [4:Lemma 1.1], and furthermore, there exist matrices $\left(A_{i k}\right),\left(a_{i k}\right),\left(B_{p_{1}}\right)$ and $\left(b_{p_{1}}\right)$ such that

$$
\begin{array}{ll}
x_{i}^{\prime}=\sum_{k=1}^{n} A_{k i} x_{k}, \quad y_{i}^{\prime}=\sum_{k=1}^{n} a_{i k} y_{k}, \quad \text { and } \quad\left(A_{i k}\right)\left(a_{i k}\right)=1_{n} ; \\
\boldsymbol{s}_{i}^{\prime}=\sum_{p=1}^{m} B_{p:} s_{p}, \quad t_{j}^{\prime}=\sum_{p=1}^{m} b_{j p} t_{p}, \quad \text { and } \quad\left(B_{p_{I}}\right)\left(b_{p q}\right)=1_{n},
\end{array}
$$

where $1_{r}$ denotes the $r \times r$ unit matrix.

Thus,

Similarly,

$$
\begin{aligned}
x_{\iota}^{\prime} s_{j}^{\prime} & =\left(\sum_{k=1}^{n} A_{k i} x_{i i}\right)\left(\sum_{p=1}^{m} B_{p j} s_{p}\right) \\
& =\sum_{k=1}^{n} \sum_{p=1}^{m} A_{k i} B_{p j} x_{k} s_{p} .
\end{aligned}
$$

$$
y_{i}^{\prime} t_{j}^{\prime}=\sum_{h=1}^{n} \sum_{q=1}^{m} a_{i h} b_{j q} y_{h l} t_{q}
$$

Therefore,

$$
\begin{aligned}
& \sum_{i=1}^{n} \sum_{j=1}^{m} \boldsymbol{x}_{\imath}^{\prime} \boldsymbol{s}_{i}^{\prime} \times \boldsymbol{y}_{\imath}^{\prime} \boldsymbol{t}_{,}^{\prime}=\sum_{h=1}^{n} \sum_{j=1}^{m}\left(\sum_{h, p=1}^{n, m} A_{k} B_{p j} \boldsymbol{x}_{k} \boldsymbol{s}_{p}\right) \times\left(\sum_{h, q=1}^{n, m} a_{i i_{k}} b_{j q} y_{h} \boldsymbol{t}_{q}\right) \\
& \simeq \sum_{i, k, h=1}^{n} \sum_{j, p, q=1}^{m}\left(A_{k i} a_{t h} B_{p i} b_{j q}\right) x_{k} s_{p} \times y_{k i} t_{q} \\
& \simeq \sum_{h=1}^{n} \sum_{p=1}^{m} x_{k} s_{p} \times y_{k} t_{p} \text {. }
\end{aligned}
$$

This proves the first part of the Lemma.

The proof of the second part is similar.

By this lemma, $A_{1} \odot A_{2}$ can be considered as a $*$-algebra with unit $1 \times 1$.

3. Norms on the space $\mathbf{A}_{1} \odot \mathbf{A}_{2}$. In this section, we consider about a norm on $A_{1} \odot A_{2}$. For any expression $\sum_{i=1}^{n} x_{i} \times y_{i} \in X$, R. Schatten defined the greatest cross-norm:

$$
\gamma\left(\sum_{i=1}^{n} x_{i} \times y_{i}\right)=\inf \left[\sum_{i=1}^{m}\left\|x_{j}^{\prime}\right\|\left|y_{j}^{\prime} \|\right| \sum_{j=1}^{m} x_{j}^{\prime} \times y_{j}^{\prime} \simeq \sum_{i=1}^{n} x_{i} \times y_{i}\right]
$$


and he proved that $\gamma(\cdot)$ has following properties :

(1) $\sum_{i=1}^{n} x_{i} \times y_{i} \simeq 0 \times 0$ if and only if $\gamma\left(\sum_{i=1}^{n} x_{i} \times y_{i}\right)=0$,

(2) $\gamma\left(\sum_{i=1}^{n} x_{i} \times y_{i}+\sum_{j=1}^{m} s_{j} \times t_{j}\right) \leq \gamma\left(\sum_{i=1} x_{i} \times y_{i}\right)+\gamma\left(\sum_{j=1}^{m} s_{i} \times t_{j}\right)$,

(3) $\gamma\left(a \cdot \sum_{i=1}^{n} x_{i} \times y\right.$. $)=|a| \cdot \gamma\left(\sum_{i=1}^{n} x_{i} \times y_{i}\right)$, where $a$ denotes a scalar,

(4) $\sum_{i=1}^{n} x_{i} \times y_{i} \simeq \sum_{j=1}^{m} s_{i} \times t_{i}$ implies $\gamma\left(\sum_{i=1}^{n} x_{i} \times y_{i}\right)=\gamma\left(\sum_{i=1}^{m} s_{j} \times t_{j}\right)$,

(5) $\gamma(x \times y)=\|x \cdot\| y \|$.

Furthermore we can prove the following:

Lemma 2. For any $\sum_{i=1}^{n} x_{i} \times y_{i}$, and $\sum_{j=1}^{m} s_{j} \times t_{j}$ in $X$,

(6) $\gamma\left[\left(\sum_{i=1}^{n} x_{i} \times y_{i}\right) \cdot\left(\sum_{j=1}^{m} s_{j} \times t_{j}\right)\right] \leq \gamma\left(\sum_{i=1}^{n} x_{i} \times y_{i}\right) \cdot \gamma\left(\sum_{j=1}^{m} s_{j} \times t_{j}\right)$,

(7) $\gamma\left[\left(\sum_{i=1}^{n} x_{i} \times y_{i}\right)\right]=\gamma\left(\sum_{i=1}^{n} x_{i} \times y_{i}\right)$.

Proof. Since (7) is similarly proved, we prove (6) only.

$$
\begin{aligned}
& \gamma\left[\left(\sum_{i=1}^{n} x_{i} \times y_{j}\right)\left(\sum_{j=1}^{m} s_{i} \times t_{j}\right)\right]=\inf \left[\sum_{k=1}^{p}\left\|u_{i k}\right\|\left\|v_{k}\right\| \sum_{h=1}^{p} u_{k} \times v_{k}^{\prime} \simeq \sum_{i=1}^{n} \sum_{j=1}^{m} x_{i} s_{j} \times y_{i} t_{j}\right] \\
& \leq \inf \left[\sum_{k=1}^{p} \sum_{h=1}\left\|x_{k}^{\prime}\right\|\left\|y_{k}^{\prime}\right\| s_{h}^{\prime}\left\|t_{h}^{\prime}\right\| \mid \sum_{k=1} x_{k}^{\prime} \times y_{k}^{\prime} \simeq \sum_{i=1}^{n} x_{i} \times y_{i}, \sum_{h=1}^{m} s_{h}^{\prime} \times t_{h}^{\prime} \simeq \sum_{j=1}^{m} s_{j} \times t_{j}\right] \\
& =\inf \left[\sum_{k=1}^{p}\left\|x_{k}^{\prime}\right\|\left\|y_{h}^{\prime}\right\| \sum_{h=1} s_{h}^{\prime}\|\| t_{h}^{\prime} \| \ldots \ldots \ldots \ldots\right] \\
& =\inf \left[\sum_{h=1}^{p}\left\|x_{k}^{\prime}\right\|\left\|y_{h}^{\prime}\right\| \mid \sum_{k=1}^{p} x_{h}^{\prime} \times y_{k}^{\prime} \simeq \sum_{i=1}^{n} x_{i} \times y_{i}\right] \\
& \quad \times \inf \left[\sum_{h=1}\left\|s_{h}^{\prime}\right\|\left\|t_{h}^{\prime}\right\| \mid \sum_{h=1}^{q} s_{h}^{\prime} \times t_{h}^{\prime} \simeq \sum s_{j} \times t_{j}\right] \\
& =\gamma\left(\sum_{i=1}^{n} x_{i} \times y_{i}\right) \cdot \gamma\left(\sum_{j=1}^{m} s_{i} \times t_{j}\right) .
\end{aligned}
$$

Now, if we consider a non-complete normed space $A_{1} \odot A_{2}$ with norm $\gamma$, we obtain a non-complete normed $*$-algebra by the properties (1)-(7) of $\gamma$; we denote this $*$-algebra by $A_{1} \odot_{\gamma} A_{2}$.

Next, we consider a linear functional $\varphi \times \psi$ on the space $A_{1} \odot A_{2}$, 
where $\phi$ and $\psi$ denote the states on $A_{1}$ and $A_{2}$, respectively. That $\phi \times \psi$ is considered as a functional on $A_{1} \odot A_{2}$; that is

$$
[\varphi \times \psi]\left(\sum_{i=1}^{n} x_{i} \times y_{i}\right)=\sum_{i=1}^{n} \phi\left(x_{i}\right) \psi\left(y_{i}\right)
$$

is invariant under the equivalence $\simeq$, is easily verified. We shall prove the following two essential lemmas.

Lemma 3. $\varphi \times \psi$ is a state on $A_{1} \odot A_{2}:$ that is, $\varphi \times \psi$ is a positive type functional with $[\varphi \times \psi](1 \times 1)=1$.

PROOF. It is sufficient to prove the following inequality:

$$
[\varphi \times \psi]\left(\left(\sum_{i=1}^{n} x_{i} \times y_{i}\right)\left(\sum_{i=1}^{n} x_{i} \times y_{i}\right)^{*}\right)=\sum_{i, j=1}^{n} \varphi\left(x_{i} x_{i}^{*}\right) \psi\left(y_{i} y_{j}^{*}\right) \geq 0
$$

for any expression $\sum_{i=1}^{n} x_{i} \times y_{i} \in X$.

Now, we recall I. E. Segal's results (cf. [3]); by his theorem, for states $\phi, \psi$, there correspond the representations of $A_{1}$ and $A_{2}$ respectively; we denote this representation spaces by $A_{1}^{\varphi}=\left\{x^{\varphi} \mid x \in A_{1}\right\}$ and $A_{2}^{\psi}=\left\{y^{\psi} \mid y \in A_{2}\right\}$, and their inner products by $\left\langle., .>_{\varphi}\right.$ and $\left\langle., .>_{\psi}\right.$ respectively. Then,

$$
\begin{array}{r}
{[\boldsymbol{\varphi} \times \psi]\left(\left(\sum_{i=1}^{n} x_{i} \times y_{i}\right)\left(\sum_{i=1}^{n} x_{i}^{*} \times y_{i}^{*}\right)\right)=\sum_{i, j=1}^{n} \phi\left(x_{i} x_{j}^{*}\right) \psi\left(y_{i} y_{j}^{* *}\right)} \\
=\sum_{i, j=1}^{n}\left\langle x_{i}^{\rho}, x_{i}^{\varphi}>_{\varphi}<y_{i}^{\psi}, y_{i}^{\psi}>_{\psi}=<\sum_{i=1}^{n} x_{i}^{\varphi} \times y_{i}^{\psi}, \sum_{j=1}^{n} x_{j}^{\varphi} \times y_{j}^{\psi}>_{\sigma}\right. \\
\text { in } A_{1}^{\varphi} \odot_{\sigma} A_{2}^{\psi},
\end{array}
$$

where $A_{1}^{\varphi} \bigodot_{\sigma} A_{2}^{\psi}$ is used in Murray-Neumann's sense [4]. Therefore $[\varphi \times \psi]\left(\left(\sum_{i=1}^{n} x_{i} \times y_{i}\right)\left(\sum_{i=1}^{n} x_{i} \times y_{i}\right)^{*}\right) \leq 0$, as desired.

Lemma 4. The set $S=\{\phi \times \psi\}$, where $\varphi$ and $\psi$ are pure states on $A_{1}$ and $A_{2}$ respectively, is complete on $A_{1} \odot A_{2}$ in the following sense: For any expression $\sum_{i=1}^{n} x_{i} \times y_{i} \neq 0 \times 0$, there exists $\varphi \times \psi$ in $S$ such that

$$
[\boldsymbol{\varphi} \times \psi]\left(\left(\sum_{i=1}^{n} x_{i} \times y_{i}\right)\left(\sum_{i=1}^{n} x_{i} \times y_{i}\right)^{*}\right)>0 .
$$

Proof. Without loss of generality, we can assume that $\left\{x_{i}\right\}$ are linearly independent. Since $A_{2}$ is a $C^{*}$-algebra, there exists a pure state $\psi$ on $A_{2}$ such that $\psi\left(y_{1} y_{1}^{*}\right)>0$.

Now, let $\bar{y}_{1}^{\psi}=y_{1}^{\psi}(\neq 0), \bar{y}_{2}^{\psi}, \ldots, \bar{y}_{k}^{\psi}$ be linearly independent elements among the elements $y_{1}^{\psi}, \ldots, y_{i}^{\psi}$ of $A_{2}^{\psi}$, and furthermore let 
ON THE DIRECT-PRODUCT OF OPERATOR ALGEBRAS

247

$$
y_{i}^{\psi}=\sum_{p=1}^{k} a_{i p} \bar{y}_{p}^{\psi} \quad i=1,2, \ldots, n
$$

be their representations by the base $\left\{\hat{y}_{p}^{\psi}\right\}$. Now, define the element $x$ in $A_{1}$ :

$$
x=x_{1}+a_{21} x_{2}+\ldots+a_{n 1} x_{n}
$$

then by the linearly independency of $\left\{x_{i}\right\}$, there exists a pure state $\varphi$ on $A_{1}$ such that $\varphi\left(x x^{*}\right)>0$. Then

$$
\begin{aligned}
& {[\varphi \times \psi]\left(\left(\sum_{i=1}^{n} x_{i} \times y_{i}\right)\left(\sum_{i=1}^{n} x_{i} \times y_{i}\right)^{*}\right)=\sum_{i, j=1}^{n} \varphi\left(x_{i} x_{j}^{*}\right) \psi\left(y_{i} y_{j}^{*}\right)} \\
& =\sum_{i, j=1}^{n}\left\langle x_{i}^{\varphi}, x_{j}^{\varphi}>_{\varphi}<y_{i}^{\psi}, y_{j}^{\psi}>_{\psi}=<\sum_{i=1}^{n} x_{i}^{\varphi} \times y_{i}^{\psi}, \sum_{,=1}^{n} x_{j}^{\varphi} \times y_{j}^{\psi}>_{\sigma}\right.
\end{aligned}
$$

in $A_{1}^{\varphi} \bigodot_{\sigma} A_{2}^{\psi}$.

Therefore, if we can prove $\sum_{i=1}^{n} x_{i}^{\varphi} \times y_{i}^{\mu} \neq 0 \times 0$ in $A_{1}^{\varphi} \odot A_{2}^{\psi}$, then the righthand side of the last equation is positive, consequently the proof is completed.

Now,

$$
\begin{aligned}
& \sum_{i=1}^{n} x_{i}^{\varphi} \times y_{i}^{\psi}=x_{1}^{\varphi} \times y_{1}^{\psi}+\cdots+x_{n}^{\varphi} \times y_{n}^{\psi} \\
& \simeq x_{1}^{\varphi} \times \overline{y_{1}^{\psi}}+x_{2}^{\varphi} \times\left(\sum_{p=1}^{k} a_{2 p} \bar{y}_{p}^{\psi}\right)+\cdots+x_{n}^{\varphi} \times\left(\sum_{p=1}^{k} a_{i n} \bar{y}_{p}^{\psi}\right) \\
& \simeq\left(x_{1}^{\varphi}+a_{21} x_{2}^{\varphi}+\cdots+a_{n 1} x_{n}^{\varphi}\right) \times \overline{y_{1}^{\psi}}+\left(a_{222} x_{2}^{\varphi}+\cdots+a_{n: 2} x_{n}^{\varphi}\right) \times \overline{y_{2}^{\psi}} \\
& \simeq x^{\varphi} \times \overline{y_{1}^{\psi}}+\cdots+\left(a_{n k} x_{n}^{\varphi}\right) \times \bar{y}_{h}^{\psi} .
\end{aligned}
$$

Since $\overline{y_{1}^{\psi}}, \ldots, \overline{y_{k}^{\psi}}$ are linearly independent in $A_{2}^{\psi}$, and $x^{\varphi} \neq 0$ in $A_{1}^{\varphi}$, consquently $\sum_{i=1}^{n} x_{i}^{\varphi} \times y_{i}^{\psi} \neq 0 \times 0$ by $[4:$ Lemma 1.1$]$ q.e.d.

Now, let $\subseteq$ be the set of positive type functional $\Phi$ such that

$$
\Phi\left(\sum_{j=1}^{m} s_{j} \times t_{j}\right)=\frac{[\varphi \times \psi]\left(\left(\sum_{i=1}^{n} x_{i} \times y_{i}\right)\left(\sum_{i=1}^{m} s_{j} \times t_{j}\right)\left(\sum_{i=1}^{n} x_{i} \times y_{i}\right)^{*}\right)}{[\varphi \times \psi]\left(\left(\sum_{i=1}^{n} x_{i} \times y_{i}\right)\left(\sum_{i=1}^{n} x_{i} \times y_{i}\right)^{*}\right)}
$$

where $\varphi \times \psi \in S$, and $\sum_{i=1}^{n} x_{i} \times y_{i}$ is an arbitrary element of $A_{1} \odot A_{2}$, and we introduce a new norm in $A_{1} \odot A_{2}$ :

(*) $\alpha\left(\sum_{i=1}^{n} x_{i} \times y_{i}\right)=\sup \left[\Phi\left(\left(\sum_{i=1}^{n} x_{i} \times y_{i}\right)\left(\sum_{i=1}^{n} x_{i} \times y_{i}\right)^{*}\right)^{1 / 2} \mid \Phi \in \subseteq\right]$, 
then we can prove the following:

LEMMA 5. The functional on $X, \alpha\left(\sum_{i=1}^{n} x_{i} \times y_{i}\right)$ defined by (*) has the following properties, (that is $\alpha(\cdot)$ is a cross-norm on $A_{1} \odot A_{2}$ in Schatten's sense [4 : Chap. II, §2]):

$$
\begin{aligned}
& \alpha\left(a \cdot \sum_{i=1}^{n} x_{i} \times y_{i}\right)=|a| \alpha\left(\sum_{i=1}^{n} x_{i} \times y_{i}\right) \text { where a denotes a scalar; } \\
& \alpha\left(\sum_{i=1}^{n} x_{i} \times y_{i}\right) \geq 0 ; \quad \alpha(1 \times 1)=1 \\
& \sum_{i=1}^{n} x_{i} \times y_{i} \simeq \sum_{j=1}^{m} s_{j} \times t_{j} \text { implies } \alpha\left(\sum_{i=1}^{n} x_{i} \times y_{i}\right)=\alpha\left(\sum_{j=1}^{m} s_{j} \times t_{j}\right), \\
& \alpha\left(\sum_{i=1}^{n} x_{i} \times y_{i}+\sum_{i=1}^{m} s_{j} \times t_{j}\right) \leq \alpha\left(\sum_{i=1}^{n} x_{i} \times y_{i}\right)+\alpha\left(\sum_{j=1}^{m} s_{j} \times t_{j}\right) \\
& \alpha\left(\left(\sum_{i=1}^{n} x_{i} \times y_{i}\right)\left(\sum_{j=1}^{m} s_{j} \times t_{j}\right)\right) \leq \alpha\left(\sum_{i=1}^{n} x_{i} \times y_{i}\right) \alpha\left(\sum_{i=1}^{m} s_{j} \times t_{j}\right), \\
& \alpha\left(\left(\sum_{i=1}^{n} x_{i} \times y_{i}\right)^{*}\right)=\alpha\left(\sum_{i=1}^{n} x_{i} \times y_{i}\right), \\
& \alpha\left(\left(\sum_{i=1}^{n} x_{i} \times y_{i}\right)\left(\sum_{i=1}^{n} x_{i} \times y_{i}\right)^{*}\right)=\left(\alpha\left(\sum_{i=1}^{n} x_{i} \times y_{i}\right)\right)^{2} \\
& \alpha\left(\sum_{i=1}^{n} x_{i} \times y_{i}\right)=0 \text { is equivalent to } \sum_{i=1}^{n} x_{i} \times y_{i} \simeq 0 \times 0 \\
& \alpha(x \times y)=\left\|x_{\|}\right\| \| y \mid .
\end{aligned}
$$

Proof. Although the argument is similar to that of Fukamiya's paper [2], we give a sketch of proof. In the course of the proof we denote $\sum_{i=1}^{n} x_{i} \times y_{i}, \sum_{j=1}^{m} s_{j} \times t_{i}$ by $\xi, \eta$ respectively, for convenience.

Ad. (8): Except the last one, all parts are clear. On the other hand, the last one follows from [4].

Ad. (9): By the definition of $\alpha(\cdot)$, for any positive $\varepsilon$ there exists a $\Phi \in \subseteq$ such that

$$
\begin{aligned}
\alpha^{2}(\xi+\eta) & -\varepsilon<\Phi\left((\xi+\eta)(\xi+\eta)^{*}\right) \\
& =\Phi\left(\xi \xi^{*}\right)+2 \operatorname{Re} \Phi\left(\xi \eta^{*}\right)+\Phi\left(\eta \eta^{*}\right) \leq \alpha^{2}(\xi)+2 \alpha(\xi) \alpha(\eta)+\alpha^{2}(\eta) \\
& =(\alpha(\xi)+\alpha(\eta))^{2} .
\end{aligned}
$$

This proves (9), since $\varepsilon$ is arbitrary.

Ad. (10): Put $\Psi(\eta)=\Phi\left(\xi \eta \xi^{*}\right) / \Phi\left(\xi \xi^{*}\right)$ for $\Phi \in \mathcal{S}$ then $\Psi(\cdot) \in \mathcal{S}$, so $\Psi\left(\eta \eta^{*}\right) \leq \alpha^{2}(\eta)$. Therefore by the definition of $\alpha(\cdot)$ 


$$
\alpha(\xi \eta) \leq \alpha(\xi) \alpha(\eta)
$$

Ad. (11): For any $\varepsilon>0$, there exists a $\Phi \in \subseteq$ such that

$$
\alpha=(\xi)-\varepsilon<\Phi\left(\xi \xi^{*}\right) \text {. }
$$

Put again $\Psi(\eta)=\Phi\left(\xi \eta \xi^{*}\right) / \Phi\left(\xi \xi^{*}\right)$, then $\Psi(\cdot) \in$ S. Therefore,

$$
\alpha^{2}\left(\xi^{*}\right) \geq \Psi\left(\xi^{*} \xi\right)=\Phi\left(\xi \xi^{*} \xi \xi^{*}\right) / \Phi\left(\xi \xi^{*}\right) \geq \Phi\left(\xi \xi^{*}\right)>\alpha^{2}(\xi)-\varepsilon .
$$

Since $\varepsilon$ is arbitrary, $\alpha\left(\xi^{*}\right) \geq \alpha(\xi)$.

By the symmetry, $\alpha\left(\xi^{*}\right)=\alpha(\xi)$.

Ad. (12): Since $\alpha\left(\xi \xi^{*}\right) \leq \alpha(\xi) \alpha\left(\xi^{*}\right)=\alpha(\xi)^{2}$ by (10), (11), it is sufficient to show $\alpha\left(\xi \xi^{*}\right) \geq \alpha(\xi)^{2}$.

For arbitrarily given $\varepsilon>0$, choose $\Phi \in \subseteq$ such that

$$
\alpha^{2}(\xi)-\varepsilon<\Phi\left(\xi \xi^{*}\right) \text {. }
$$

Then,

$$
\left(\alpha^{2}(\xi)-\varepsilon\right)^{2}<\Phi\left(\xi \xi^{*}\right)^{2} \leq \Phi\left(\xi \xi^{*} \xi \xi^{*}\right) \leq \alpha^{2}\left(\xi \xi^{*}\right) .
$$

Therefore $\alpha^{2}(\xi) \leq \alpha\left(\xi \xi^{*}\right)$ by the arbitrariness of $\varepsilon$.

Ad. (13): This immediately follows from Lemma 4.

Ad. (14): Firstly we shall prove $\alpha(\xi) \leq \gamma\left(\xi \xi^{*}\right)^{1 / 2}$. Indeed, for any real number $k>\gamma\left(\xi \xi^{*}\right)$, the element

$$
\xi^{\prime}=\left(1 \times 1-\frac{\xi \xi^{k}}{k}\right)^{1 / 2}
$$

exists in the complete $*$-algebra $A_{1} \times_{\gamma} A_{2}$ (=the completion of $A_{1} \odot_{\gamma} A_{2}$ by $\gamma(\cdot))$, and $\xi^{\prime}=\xi^{* *}$. Therefore for any $\Phi \in \mathfrak{S}$,

$$
0 \leq \Phi\left(\xi^{\prime} \xi^{*}\right)=\Phi\left(\xi^{\prime 2}\right)=\Phi\left(1 \times 1-\frac{1}{k} \xi \xi^{*}\right)=1-\frac{1}{k} \Phi\left(\xi^{*}\right),
$$

Consequently,

$$
\Phi\left(\xi \xi^{*}\right) \leq \gamma\left(\xi \xi^{*}\right)
$$

Then by the definition of $\alpha(),. \alpha(\xi) \leq \gamma\left(\xi \xi^{*}\right)^{1 / 2}$.

Using this fact,

$$
\alpha(x \times y) \leq \gamma\left((x \times y)(x \times y)^{*}\right)^{1 / 2}=\left(\left\|x x^{*}\right\|\left\|y y^{*}\right\|\right)^{1 / 2}=\|x\| \cdot|y| .
$$

On the other hand,

$$
\begin{aligned}
\alpha(x \times y) & =\sup \left[\Phi\left(x x^{*} \times y y^{*}\right)^{1 / 2} \mid \Phi \in \Xi\right] \geq \sup \left[\left(\varphi\left(x x^{*}\right) \psi\left(y y^{*}\right)\right)^{1 / 2} \mid \varphi \times \psi \in S\right] \\
& =\|x\| \cdot\|y\| .
\end{aligned}
$$

This completes the proof.

From the above considerations, $A_{1} \odot_{\alpha} A_{2}$ is a non-complete $C^{*}$-algebra with unit $1 \times 1$, so completing $A_{1} \odot_{\alpha} A_{2}$, we obtain a new $C^{*}$-algebra, and we denote this algebra by $A_{1} \times_{\infty} A_{2}$. Thus the following main theorem has been completely proved.

THEOREM 1. Let $A_{1}$ and $A_{2}$ be any $C^{+}$-algebras (with unit 1), and define the cross-norm $\alpha(\cdot)$ on the direct product *algebra $A_{1} \odot A_{2}$ by $\left(^{*}\right)$, then the 
completion $A_{1} \times_{a} A_{2}$ is also a $C^{*}$-algebra (with unit).

4. Direct-product of commutative algebras. In this section, we consider the commutative case which may justify our definition of norm on the direct-product.

Let $\Omega$ and $\Gamma$ be compact Hausdorff spaces and, $C(\Omega)$ and $C(\Gamma)$ be the $C^{*}$-algebras of all complex-valued continuous functions on the space $\Omega$ and $\Gamma$ with usual norm and involution, respectively. Then we can prove the following :

Theorem 2. $C(\Omega) \times{ }_{n} C(\Gamma)$, the direct-product of $C(\Omega)$ and $C(\Gamma)$ in the sense of Theorem 1, can be represented isometricaily isomorphic as the $C^{*}$-algebra of all continuous functions on the product space $\Omega \times \Gamma$.

LEMMA 6. The mapping $\sum_{i=1}^{n} x_{i} \times y_{i} \rightarrow \sum_{i=1}^{n} x_{i}(\cdot) y_{i}(\cdot)$ is the isometric homomorphism from $C(\Omega) \odot_{a} C(\Gamma)$ into the $C(\Omega \times \Gamma)$.

ProOF. Firstly we prove this mapping is invariant under the equivalence $\simeq$. Indeed, if $\sum_{i=1}^{n} x_{i} \times y_{i} \simeq \sum_{j=1}^{m} s_{i} \times t_{j}$, then without loss of generality, we can assume that $\left\{x_{i}\right\}, \ldots,\left\{t_{j}\right\}$ are linearly independent respectively, and $n=m$, furthermore there exist matrices $\left(A_{i j}\right)$ and $\left(a_{i j}\right)$ such that

$$
s_{k j}=\sum_{j=1}^{n} A_{i k} x_{j}, \quad t_{k}=\sum_{j=1}^{n} a_{i, j} y_{j}, \text { and }\left(A_{j k}\right)\left(a_{i k}\right)=1_{n} .
$$

Then,

$$
\begin{aligned}
\sum_{k=1}^{n} s_{h}(\cdot) t_{k}(\cdot) & =\sum_{h=1}^{n}\left(\sum_{j=1}^{n} A_{j k} x_{j}(\cdot)\right)\left(\sum_{h=1}^{n} a_{k, h} y_{h}(\cdot)\right) \\
& =\sum_{k=1}^{n} \sum_{j, i=1}^{n} A_{j k} a_{k \prime \prime l} x_{j}(\cdot) y_{i}(\cdot)=\sum_{j=1}^{n} x_{j}(\cdot) y_{j}(\cdot) .
\end{aligned}
$$

Since that this mapping is a homomorphism is clear, it is sufficient to show the isometric property. While by the definition of $\alpha(\cdot)$,

$$
\begin{aligned}
\alpha\left(\sum_{i=1}^{n} x_{i} \times y_{i}\right) & =\sup \left[[\omega \times \gamma]\left(\left(\sum_{i=1}^{n} x_{i} \times y_{i}\right)\left(\sum_{i=1}^{n} x_{i} \times y_{i}\right)^{*}\right)^{1 / 2} \mid \omega \in \Omega, \gamma \in \Gamma\right] \\
& =\sup \left[\left(\sum_{i, j=1}^{n} x_{i} x_{j}^{*}(\omega) y_{i} y_{j}^{*}(\jmath)\right) 1 / 2 \mid \omega \in \Omega, \gamma \in \Gamma\right] \\
& =\sup \left[\left|\sum_{i=1}^{n} x_{i}(\omega) y_{i}(\gamma)\right| \mid \omega \in \Omega, \gamma \in \Gamma\right] \\
& =\left\|\sum_{i=1}^{n} x_{i}(\cdot) y_{i}(\cdot)\right\| \quad \text { in } C(\Omega \times \Gamma) .
\end{aligned}
$$


Proof of Theorem 2. By the above Lemma $6, C(\Omega) \times_{\alpha} C(\Gamma)$ is embeded in $C(\Omega \times \Gamma)$ by the isometrically isomorphic mapping, so the image $R$ of $C(\Omega) \times{ }_{\alpha} C(\Gamma)$ is a closed *-subring of $C(\Omega \times \Gamma)$. Furthermore for any distinct points $\left(\omega_{1}, \gamma_{1}\right)$ and $\left(\omega_{2}, \gamma_{2}\right)$ there exists clearly an element $r$ of $R$ such that $r\left(\omega_{1}, \gamma_{1}\right) \neq r\left(\omega_{2}, \gamma_{2}\right)$, so $R=C(\Omega \times \Gamma)$.

REMARK. Concerning the algebraic properties of the direct-productfor example, simplicity, factoriality of $A_{1} \times{ }_{a} A_{2}$-we shall discuss in the later paper in this journal.

\section{REFERENCES}

[1] N. Dunford-R. SCHATTEN, On the associate and conjugate spaces for the direct product, Trans. Amer. Math. Soc., 59(1946), 430-436.

[2] M Fukamiya, On B*-Algebras, Proc. Japan Acad., Tokyo 27(1951), 321-327; and, On a theorem of Gelfand and Neumark and the B*-algebra, Kumamoto Journ. of Sci., 1(1952), 17-22.

[3] I. E.SEGAL, Irreducible representations of operator algebras, Bull. Amer. Math. Soc., 53(1947), 73-88.

[4] R. SchATTEN, Theory of cross-spaces, Princeton (1950).

Mathematical Institute, TôHoku University, SendaI. 